DOI: https://doi.org/10.34069/AI/2021.45.09.2

How to Cite:

Makhachashvili, R., Bakhtina, A., \& Semenist, I. (2021). La función de la inteligencia emocional en la educación digital como el sustrato de la validez de la vida on-line. Amazonia Investiga, 10(45), 20-30. https://doi.org/10.34069/AI/2021.45.09.2

\title{
La función de la inteligencia emocional en la educación digital como el sustrato de la validez de la vida on-line
}

\section{Функція емоційного інтелекту в цифровій освіті як субстрат валідації онлайн-буття}

Received: August 10, 2021

\section{Resumen}

La Industria 4.0, cuyo sustrato es la introducción de la matriz ciberfísica en la producción mundial, ha realizado importantes ajustes en el sistema de los valores humanos, lo que ha llevado a una reorientación de la esencia ontológica de la humanidad. Esto afectó principalmente la misión y la visión de la educación. La reorientación se refiere a la transgresión de la "vida real" al formato digital. Partiendo de lo expuesto, nuestra investigación tiene como objetivo identificar la función y la importancia del funcionamiento de la inteligencia emocional en la educación digital como una condición previamente necesaria para el proceso evolutivo en el mundo. Con el motivo de explicar el núcleo del problema del funcionamiento de la inteligencia emocional en la educación digital, se realizó una encuesta empírica de los participantes en el proceso educativo (los estudiantes), que permitió inferir un modelo hipotético-deductivo de la percepción emocional del ser en línea en sus varios formatos. El modelo se presenta aplicando un enfoque lógico-praxiológico (el principio de la explosión con la verificación mediante la tabla de verdad) y consiste en la contradicción entre el ser-ahí determinado físicamente y el ser en línea previsto tecnológicamente con la correspondiente transgresión de la actividad humana del Dasein al deadline.
Accepted: September 23, 2021

Written by:

Rusudan Makhachashvili ${ }^{4}$ https://orcid.org/0000-0002-4806-6434

Anna Bakhtina ${ }^{5}$

https://orcid.org/0000-0003-3337-6648

Ivan Semenist ${ }^{6}$

https://orcid.org/0000-0002-0847-8856

\begin{abstract}
Анотація
Індустрія 4.0, субстратом якої $\epsilon$ впровадження кіберфізичної матриці у світове виробництво, внесла важливі корективи в систему цінностей людини, що зумовило переорієнтацію онтологічної сутності людства. Першочергово це відобразилося на місії та візії освіти. Переорієнтація стосується трансгресії “фактичного життя” у цифровий формат. Таким чином, дослідження спрямоване на виявлення ролі та важливості функціонування емоційного інтелекту в цифровій освіті як передумові еволюційного процесу в світі. 3 метою експлікації проблемного ядра функціонування емоційного інтелекту в цифровій освіті проведено емпіричне опитування учасників освітнього процесу (здобувачів освіти), що дозволило вивести гіпотетико-дедуктивну модель емоційної перцепції on-line-буття в різних його форматах. Модель подається із застосуванням логіко-праксіологічного підходу (логічний принцип вибуху 3 верифікацією за допомогою таблиці істинності) і полягає в наявності протиріччя між фізикалістськи зумовленим тут-буттям та технологічно передбаченим on-line-буттям 3 відповідною трансгресією людської діяльності з Dasein y deadline.
\end{abstract}

\footnotetext{
${ }^{4}$ Doctor habilitado, Profesor Asociado University de Kyiv Borys Grinchenko, Ucrania.

${ }^{5}$ Estudiante de posgrado, Profesor Asistente University de Kyiv Borys Grinchenko, Ucrania.

${ }^{6} \mathrm{PhD}$ University de Kyiv Borys Grinchenko, Ucrania.
} 


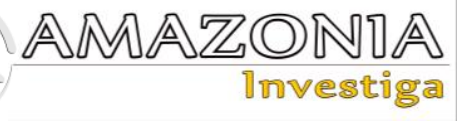

Palabras clave: inteligencia emocional, educación digital, vida on-line, el ser-ahí, educación a distancia.

\section{Introducción}

La pandemia del Covid-19 hizo ajustes espontáneos a la dimensión de la existencia humana (Makhachashvili et al, 2020a), (DQ Global Standards Report, 2019), que adiaforiza la existencia fisica, transgrediéndola al ser en línea como el único ser permitido. El ser en línea es esencialmente una versión del ser-ahí (Heidegger y Hofstadter, 1988), ya que M. Heidegger enfatizó dos posiciones opuestas al mismo tiempo: por un lado, dice que el hombre moderno es tan conocido como nunca lo había sido antes. Por otro lado, el hombre mismo, según el filósofo, es más problemático que nunca. Todo esto se explica por la conclusión de Heidegger sobre la "disposición ontológica del hombre moderno" (Heidegger y Hofstadter, 1988, pág. 19). El siglo XXI ha transformado el concepto de este filósofo al subordinarlo a las condiciones de la cuarentena mundial. La búsqueda de la esencia existencial del hombre consiste en "captar explícitamente la movilidad definitoria de la vida actual" (Heidegger \& Hofstadter, 1988, pp. 241), que determina el serahí con una visualización instantánea de la vida. En primer lugar, esta visualización se refiere a uno de los factores principales que posibilita la evolución humana: la educación. Dado que la pandemia ha provocado una localización digital absoluta del espacio educativo, una condición necesaria es la introducción del concepto del ser en línea, en el que el Dasein (Heidegger \& Hofstadter, 1988, pp.200) es relevante para el sustrato de la validez de la educación a distancia, cuyo concepto central es "deadline". El ser en línea, a su vez, se caracteriza por una disonancia cognitiva (Damasio, 1994), que consiste en la colisión de las dos dimensiones opuestas: el infinito digital y la existencia física finita. Todo esto se refleja en la educación digital: el comunicante digital no solo debe validar sus actividades prácticas en la red, sino también aprender a transmitir emociones en el formato digital con el fin de adaptarse a la situación (la vida real) y estar en contacto con otro comunicante lo que sintetizará la posibilidad de evitar las barreras de la comunicación (Hymes, 1972), que son el sustrato de los conflictos, las colisiones, las guerras en la red, etc. y así promoverá la educación eficaz en el formato a distancia.

Nuestra tarea es explicar el núcleo problemático del funcionamiento de la inteligencia emocional
Ключові слова: емоційний інтелект, цифрова освіта, on-line-буття, тут-буття, дистанційне навчання.

en la educación digital, que, de hecho, visualiza la actitud del participante del proceso educativo hacia el formato de la educación a distancia que se realiza por las competencias cualitativas, válidas para la educación digital en el siglo XXI (Makhachashvili et al, 2020b), (Davies, Fidler et al, 2011), (Dos Reis, 2015). La encuesta empírica de los participantes en el proceso educativo (los profesores y los estudiantes) derivará en un modelo hipotético-deductivo de la percepción del ser en línea en sus formatos diversos, que se concluirá en una variante de un silogismo hipotético, cuyo significado verdadero es desconocido. Lo último verificará el campo problemático del funcionamiento ineficiente de la inteligencia emocional, explicando el ser en línea como verdadero y no verdadero (según el principio de explosión). Aunque en el razonamiento deductivo el valor de la verdad se transfiere a la conclusión, en nuestro caso la base son las hipótesis, y por lo tanto la conclusión del razonamiento hipotético-deductivo será probable, pero aprobado por los sistemas lógicos, cuyas reglas consisten en que los enunciados pueden deducirse de las contradicciones. Es decir, si permitimos las contradicciones, cualquier enunciado será la consecuencia de la contradicción.

Esta investigación explica el núcleo del problema del funcionamiento de la inteligencia emocional en la educación digital. Las cuestiones de la investigación fueron las siguientes:

1. ¿Cuál es función de la inteligencia emocional en la educación digital?

2. ¿Cuál es la diferencia entre el ser-ahí y el ser en línea en la intersección de la búsqueda de la esencia existencial del hombre?

3. ¿Cuál es la contradicción principal en la percepción humana de la educación digital?

La parte empírica de la investigación, relacionada con la obtención de los datos para su posterior procesamiento analítico, se llevó a cabo mediante un cuestionario ("Herramientas y prácticas de TIC para la evaluación de la calificación final en el marco de la cuarentena por la pandemia del COVID-19") para los profesores y estudiantes de la Universidad de Kyiv Borys Grinchenko: 59 encuestados participaron en una encuesta anónima basada en el cuestionario mencionado y un análisis más detallado de los 
resultados sobre el uso de varias herramientas de TIC en la educación digital, principalmente debido a la pandemia. Los resultados más detallados en varios aspectos se presentan en un ciclo de publicaciones del grupo de autores (Makhachashvili et al, 2020a), (Makhachashvili et al, 2020b), (Makhachashvili et al, 2021c).

La información se recopiló en el marco del protocolo de ética de la investigación y de la cultura corporativa de la Universidad de Kyiv Borys Grinchenko teniendo en cuenta la confidencialidad de los encuestados con su consentimiento previo para el siguiente procesamiento de los datos analíticos. La encuesta se centra en las tecnologías digitales utilizadas en el proceso de la educación digital en particular y en la educación a distancia durante la cuarentena provocada por la pandemia del COVID-19, lo que también fomentó la activación de una gran cantidad de las herramientas digitales:

1. E-mail

2. Google Disc

3. Google Forms

4. LMS Moodle (or other learning management systems)

5. Automated testing systems (LMS)

6. Automated testing systems (online, cloud based)

7. Hang Out Meet (Google video-conferencing service)

8. Zoom (videoconferecing service)

9. Android apps

10. Microsoft Teams

11. Adobe Connect

12. Webex

13. Online radomiser

14. Screen sharing features

15. Microsoft Office tools (Word, PowerPoint, Excel spreadsheets, etc)

16. Speech to text interface

17. Cloud based presentation tools (for e.g. Prezi)

18. Social Media platforms (Facebook, Telegram, Instagram, Twitter, etc.)

La evaluación de los encuestados de la importancia de estas actividades y de la aplicación de las tecnologías mencionadas de TIC en la educación digital contribuyó a la visualización de las características perceptivas de los encuestados de las herramientas, lo que identifica su emotio en la intersección de la implicación sujeto-objetiva de la actividad en la síntesis con la ratio, transgredido al ser en línea y automatizado por él.

\section{Marco Teórico o Revisión de literatura}

La integración de la educación al espacio digital ha separado dos factores fundamentales de la esencia existencial del hombre: 1) el ratio, cuya ventaja es la racionalidad sustantiva como un sistema generalizado de valores y estándares integrados en la cosmovisión (Weber et al., 1958); 2) el emotio, que a priori sigue siendo el sustrato del proceso psíquico, que implica la adaptación del sistema nervioso central a los cambios externos, asumiendo la exactitud de la percepción subjetiva de la cosmovisión racional (Bar-On, 1988), (Boyatzis, Goleman, 2001). Sin embargo, surge una pregunta lógica: ¿Es capaz de coexistir sinérgicamente el homo ciberneticus (Restian, 1981) con estos dos factores en el mundo digital? ¿No está aislado el ratio del emotio bajo las restricciones espaciales? ¿O seguimos observando la adiaforización de las fronteras (de la raza, de la especie, etc.), localizados en el universo virtual como la sustancia única? Notemos de antemano que, en nuestra opinión, la síntesis de la amorfia digital proporcionada por el sistema no lineal del tiempo-espacio en el ser en-línea y la deformación de la comprensión clásica de la educación se extiende a la síntesis amorfodeformada de la cultura como una forma atributiva de la autoidentificación del homo ciberneticus.

Los investigadores Muhammad Ayub Buzdar, Akhtar Ali y Riaz Ul Haq Tariq intentaron ir más allá de la comprensión clásica de la educación y exploraron los aspectos psicométricos de la preparación de los estudiantes para la educación digital. La atención se centró principalmente en la función de la inteligencia emocional para explicar las diferencias, desventajas y ventajas de la educación digital: «Examinamos cuatro aspectos de la preparación de los estudiantes para la educación digital y descubrimos que los estudiantes tenían una preparación comparativamente más fuerte en la motivación para el aprendizaje y el aprendizaje autodirigido. Los estudiantes demostraron una capacidad de control casi promedio en un contexto en línea; sin embargo, la autoeficacia de la comunicación en línea y del uso del ordenador/Internet fue comparativamente inferior entre la muestra» (Buzdar et al., 2016, p. 154). Según los indicadores de la preparación de los estudiantes para la educación digital, que fluctúan en la nota media, lo que indica una disposición parcial para el aprendizaje en línea, los autores destacan la presencia de la inteligencia emocional en la educación digital como el sustrato del que dependerá la eficacia del aprendizaje: 


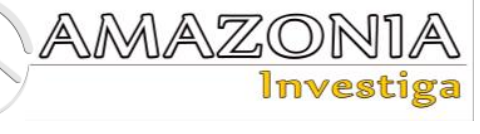

«Recomendamos hacer más investigaciones para desarrollar las estrategias istruccionales que promuevan la inteligencia emocional de los estudiantes con la intención de mejorar su preparación y el desempeño en los entornos del aprendizaje en línea» (Buzdar et al., 2016, p. 154).

A su vez, los investigadores de las comunicaciones digitales, Alexander J.A.M. van Deursen, Colin L. Bolle, Sabrina M.Hegner, Piet A.M. Kommers, según una encuesta de los 386 encuestados, dicen que el estrés social, por el contrario, tiene un efecto positivo en la adaptación humana a la vida en línea, desarrollando la competencia digital $\mathrm{y}$, en consecuencia, la inteligencia emocional en este caso se desarrolla de forma más rápida y efectiva: «Las personas que utilizan ampliamente sus teléfonos inteligentes con fines sociales desarrollan los hábitos de su uso más rápidamente, lo que a su vez puede conducir a un comportamiento adictivo respecto a los teléfonos inteligentes. No encontramos una influencia de la inteligencia emocional en el comportamiento habitual o adictivo con los teléfonos inteligentes, mientras que el estrés social influye positivamente en el comportamiento adictivo con los teléfonos inteligentes, y un fallo en la autorregulación parece causar un mayor riesgo del comportamiento adictivo con los teléfonos inteligentes» (van Deursen et al., 2015, p.419).

Se realizó una investigación exhaustiva durante la cuarentena en India (abril-mayo del año 2020), que tuvo como objetivo analizar la percepción del estrés académico que experimentan los estudiantes en la educación digital y desarrollar las estrategias para superarlo con la ayuda de la inteligencia emocional. La autora Yamini Chandra realizó una doble encuesta utilizando dos cuestionarios en línea: "Percepción de la escala de estrés académico" y "Escala de la inteligencia emocional", cuyos resultados fueron analizados mediante una prueba $\chi^{2}$. Los resultados mostraron que «Mediante el uso de la inteligencia emocional y el alejamiento del aburrimiento y los pensamientos depresivos, los estudiantes intentaban hacer frente a los efectos negativos derivados de la actual situación pandémica" (Chandra, 2020).

Así, es obvio que la percepción de los estudiantes de la educación digital difiere significativamente de la aceptación de la educación digital como una realidad del siglo XXI (el ser en línea) y su aceptación como la educación a distancia encerrada en la cuarentena por la pandemia del Covid-2019. Todo esto, a su vez, distingue las funciones y el desarrollo de la inteligencia emocional: el factor pandémico explica la falta de preparación para la educación digital, cristalizando de forma inmanente el factor del miedo; el factor de la realidad del siglo XXI, por el contrario, elimina las barreras psicológicas. De hecho, nos centraremos más en esta contradicción.

Sin embargo, el ser en linea plantea otro tema problemático, que es la discusión sobre el desarrollo de la inteligencia emocional (Davis et al, 1998), (Conte, 2005), (Keefer et al, 2018) en el espacio digital educativo: ¿qué eficiencia tiene la inteligencia emocional en la intersección de la interdependencia de la tecnología, donde el enfoque principal de los estudiantes y los profesores se traspasa al objetivo de la cámara?

La cuestión de la inteligencia emocional en la educación aparece a priori como un punto de dificultad. Los científicos enfatizan la colisión de la tradición y de la innovación en la educación, que sintetiza la inteligencia racional y emocional: "...la promoción de la inteligencia emocional en las escuelas ha demostrado una búsqueda controvertida, desafiando los puntos de vista tradicionales "racionalistas" de la educación. Además, los resultados de la investigación en esta área han sido, en el mejor de los casos, inconsistentes" (Humphrey et al., 2007, p. 245).

M. Radford deduce su propio concepto de la función de la inteligencia emocional en la educación, y concluye que hay un «Resurgimiento de dos comprensiones en la relación con una perspectiva actual sobre la naturaleza de la inteligencia emocional. La primera comprensión es de carácter dualista, viendo las emociones como eventos internos sujetos a la introspección. La segunda ve las emociones como fuentes de energía o tensión que pueden aliviarse en el proceso de la articulación, es decir, una especie de dimensión terapéutica en un contexto "confesional". Estas dos comprensiones en su conjunto forman una perspectiva que puede denominarse la hipótesis "introspectiva/confesional". Con esta hipótesis se revisan algunos de los problemas filosóficos tradicionales y se ofrece una perspectiva alternativa de las emociones. Esta perspectiva alternativa considera que nuestras vidas emocionales se desarrollan dentro de un contexto social y cultural, como esencialmente públicas y abiertas a la objetivación, lo que podría denominarse una hipótesis 'objetivista/constructivista'» (Radford, 2006, p. 227). Según el investigador, la inteligencia emocional es la función más relevante y práctica 
de la educación en la dimensión moderna, lo que es especialmente cierto en la educación digital, donde las relaciones de "sujeto-sujeto" son reemplazadas por las de "sujeto-objeto", en las cuales el actante principal ("el posibilitador") del ser en línea no es una persona, sino un deadline. Los investigadores Adel Zahed-Babelana y Mahdi Moenikia han considerado los problemas de la función de la inteligencia emocional exclusivamente en la educación a distancia. Las conclusiones de los científicos, basadas en la parte experimental, confirman que «Los dominios afectivos como las habilidades interpersonales, el manejo del estrés, la adaptabilidad y el modo general se están fusionando lentamente en los diseños instruccionales de la educación a distancia. Las características psicológicas son vistas como las características necesarias de los estudiantes eficientes en los programas de la educación a distancia y su continuidad en estos programas» (Zahed-Babelan \& Moenikia, 2010, p. 1160).

Así, partiendo de que la educación digital no se realiza en el espacio interpersonal, sino en el intrapersonal, los profesores y los estudiantes se convierten en los funcionales del actante principal del ser en línea. De lo último, a su vez, se deduce que el ser en línea es verdadero y falso al mismo tiempo, porque para un comunicante digital el deadline implica el ser en línea (para el profesor), y por lo tanto está controlado, mientras que para el otro (para el estudiante) solo se proporciona en el ser en línea, y, como consecuencia, no está guiado, sino subordinado a la vida real (al ser-ahí). En tales colisiones ontológicas se produce la verificación emocional del "hombre moderno sin disposisión", que raya en la búsqueda de uno mismo no solo en la dimensión educativa, sino también en la dimensión existencial en general: «En la educación a distancia, existe una brecha entre el profesor y el alumno, por lo que el alumno debe aceptar el grado superior de la responsabilidad en la realización del programa de aprendizaje. El alumno autónomo necesita poca ayuda del profesor, que puede ser más un respondedor que un director» (Zahed-Babelan \& Moenikia, 2010, p. 1162).

\section{Metodología}

El análisis de la investigación se basa en un enfoque lógico-praxiológico. La síntesis de ambos enfoques es una complementariedad sinérgica del plan de contenido del objeto de la investigación. El aspecto praxiológico es una forma específica de analizar la actividad humana en la explicación de su relevancia para el propósito de la acción, la racionalidad, la eficiencia, que se correlaciona con la "vida actual" del ser-ahí, transgredido en el ser en línea. Esto revela la esencia de la organización de las actividades, abarcando tres grupos de los problemas: descripción analítica, caracterización, clasificación y sistematización de las acciones prácticas, así como el estudio de las condiciones y las leyes que determinan la eficacia de las acciones. El enfoque lógico permite formalizar los datos obtenidos empíricamente, localizando los resultados en el nivel estructural (ascendente), lo que es una condición necesaria para validar el nivel fenomenológico regulado por la psicología humana, incluida la inteligencia emocional. Ante todo, la lógica es nuestra herramienta, y el principio de explosión (del latín ex falso quodlibet) reconstruirá hipotéticamente el concepto del conjunto de los mundos (el ser-ahí, el ser en-línea) y, por lo tanto, el conjunto de las verdades, lo que presupone la educación digital no como el estrés/la impotencia/la imposibilidad, sino como una regularidad de la realidad óntica del siglo XXI, normalizando así la eficacia de la inteligencia emocional.

La creación de un silogismo hipotético contribuye al establecimiento de un modelo hipotético-deductivo implícito del núcleo del problema del funcionamiento de la inteligencia emocional en la educación digital. Por analogía, comparamos el ser-ahí y el ser en línea transfiriendo las propiedades del primero al segundo, en particular en el proceso educativo, trazando así la correlación entre los dos objetos. Por extrapolación, traslademos los datos hipotéticos obtenidos a las tablas analíticas de la lógica intuicionista con la idea de establecer una proposición de cierto/falso en las premisas y en la conclusión sobre la eficacia de la inteligencia emocional en la educación digital.

\section{Resultados y discusión}

Las respuestas a la pregunta "Identifique todas las herramientas TIC que tuvo que emplear a lo largo del proceso de la evaluación digital de la calificación" mostró que todas estas herramientas son más o menos utilizadas por los encuestados en la educación digital (fig. 1) 


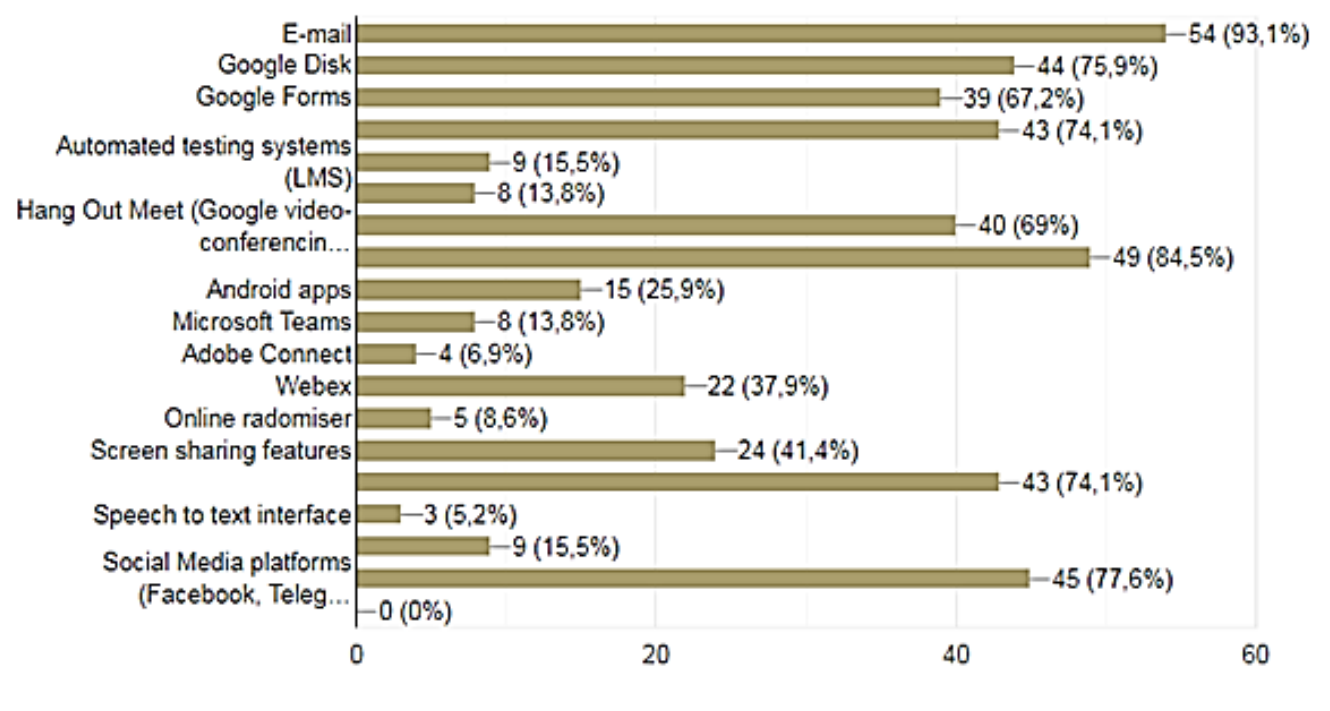

Figura 1. Herramientas TIC utilizadas por los encuestados en la educación digital. Fuente de datos: Investigación propia (Makhachashvili et al, 2021c)

Para la educación digital efectiva es importante la competencia praxiológica de un estudiante, que cuenta con tres factores para asegurar la adaptación digital: motivación (contribuye a la formación de nuevas metas y su consecución); flexibilidad (la capacidad de controlar sus estados internos, la capacidad de gestionar los cambios en el entorno social, la capacidad de actuar y tomar decisiones en el estrés); conciencia (la capacidad de asumir la responsabilidad de las tareas y su desempeño, la capacidad de disfrutar de cumplir de sus obligaciones). Todos estos factores en su conjunto codifican las especificidades de la comunicación digital, donde el concepto de la comunicación como tal pasa a primer plano (y el principal) de la autorrealización humana en el ser en línea y la aprobación de sus acciones en el tiempo real. Así, según los resultados del análisis de las respuestas a la pregunta "Identifique las habilidades blandas (soft skills) más destacadas para las herramientas TIC que tuviste que emplear a lo largo del proceso de evaluación digital de la calificación" obtenemos una ventaja de la comunicación en las siguientes tecnologías de TIC (fig. 2):

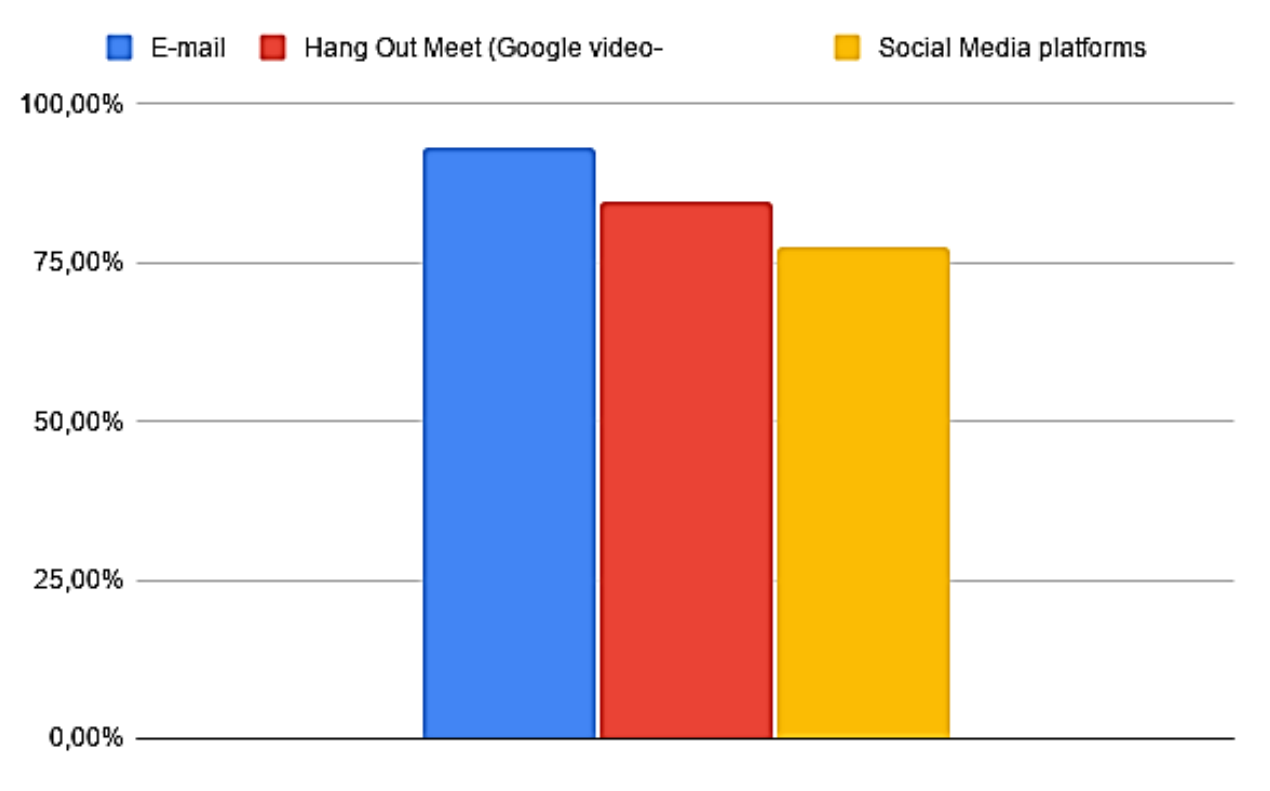

Figura 2. Una ventaja de la comunicación en las siguientes tecnologías de TIC. Fuente de datos: Investigación propia (Makhachashvili et al, 2021c) 
Si las redes sociales a priori son creadas para la introducción y el desarrollo de la comunicación digital, en el proceso educativo el correo electrónico y las herramientas de video conexión actúan como convergentes, lo que reproduce miméticamente el propósito funcional de las redes sociales con la diferencia de que la convergencia inmanente forma un sistema de uso más complejo. Bajo esta condición, el estudiante buscará en el ser en línea una salida al ser-ahí emocional, que consiste en la comprensión entre el sujeto (una persona) y el objeto (una máquina), y por tanto, la necesidad de la comunicación como una condición necesaria de la inteligencia emocional. Lo último ha predicho la selección de las TIC (fig. 3) específicas por la orientación comunicativa (sincrónica y asincrónica).

\section{Communication}

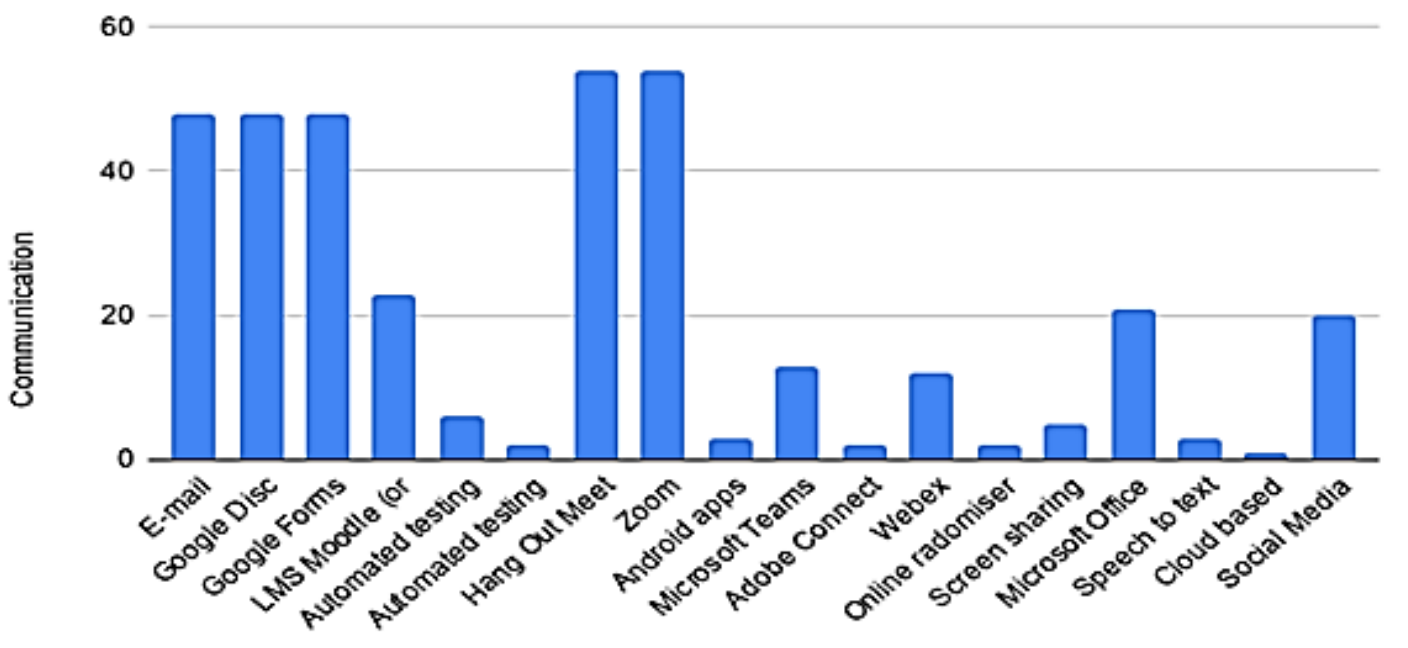

Figura 3. La selección de las TIC específicas por la orientación comunicativa (sincrónica y asincrónica). Fuente de datos: Investigación propia

Los resultados de la encuesta mostraron que la importancia de la inteligencia emocional en la educación digital supera a otras competencias, lo que asegura la existencia conjuntiva del ser-ahí y del ser en línea con "la captación explícita de la movilidad determinante de la vida real". Sin embargo, la facticidad de este último conduce a una contradicción, porque, a diferencia del Dasein, el ser en línea se caracteriza por la presencia de un deadline. Lo último, a su vez, se explica como un plazo (fecha y/o hora) límite para realizar una tarea determinada, incluida la comunicación. La contradicción se basa en la infinidad del espacio y la finitud del tiempo, lo que en muchos casos provoca un estrés académico. Sin embargo, de acuerdo con los resultados de la investigación, se concluyó que el enfoque de la educación digital en la comunicación es una validez de la necesidad de desarrollar la inteligencia emocional, que promoverá un aprendizaje efectivo. Se trata de la comunicación sincrónica y asincrónica con una ventaja natural de la sincrónica. Sin embargo, el propio hecho de la comunicación se convierte en una mimesis del actante del ser en línea (el profesor $\mathrm{y}$ el estudiante). $\mathrm{Y}$ dado que la educación digital está dirigida al espacio intrapersonal, la inteligencia emocional, a su vez, solo verifica y aumenta el valor del proceso de la autoidentificación en el ser en línea y la autorregulación de los procesos psíquicos y cognitivos (fig. 4). 


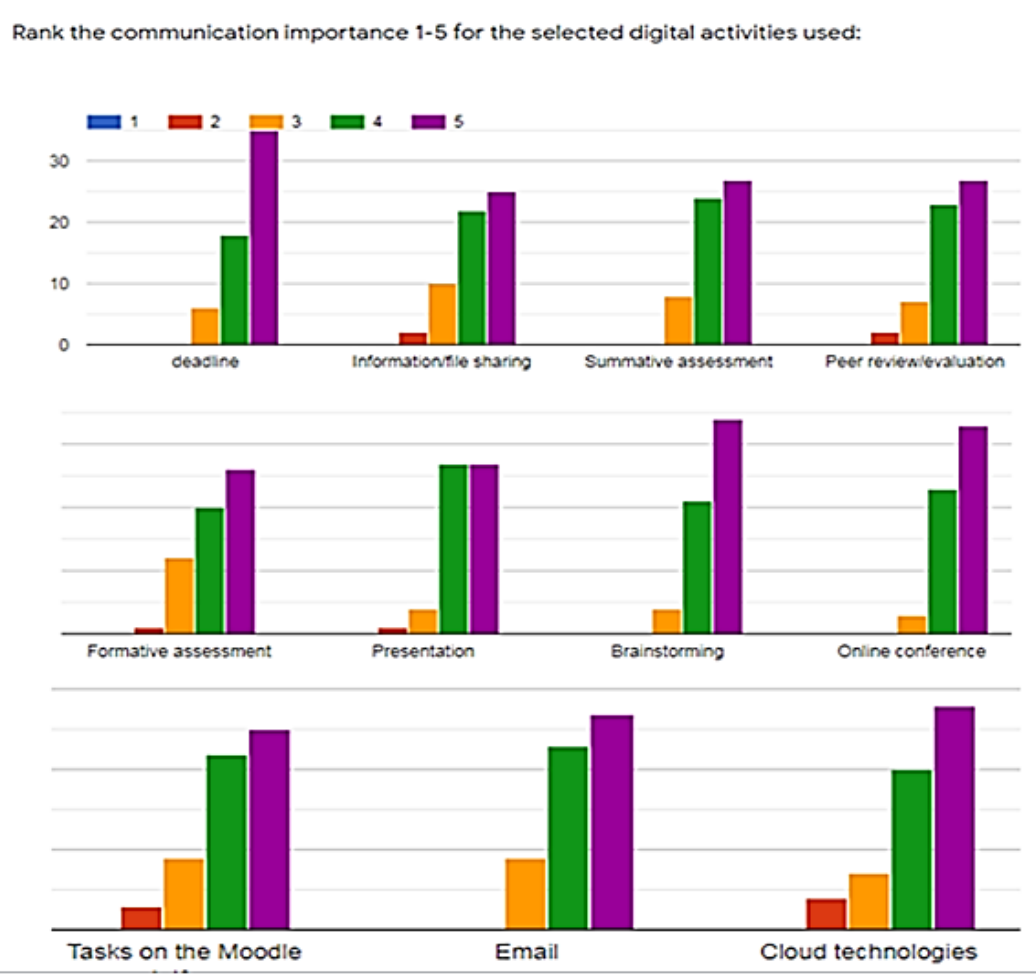

Figura 4. El enfoque de la educación digital en la comunicación.

Fuente de datos: Investigación propia (Makhachashvili et al, 2021c)

De acuerdo con los resultados de la investigación, extrapolamos datos hipotéticos a la fórmula del principio de explosión.

$$
\mathrm{A} \forall \mathrm{B}:(\mathrm{A} \cap \neg \mathrm{A}) \Rightarrow \mathrm{B}
$$

Como ya se mencionó, PE en la lógica clásica e intuicionista consiste en que cualquier enunciado puede deducirse de la contradicción. Es decir, si se identifica una contradicción, entonces cualquier enunciado y su negación serán consecuencia de la contradicción. Extrapolando el ser-ahí y el ser en línea a un conjunto (ser), podemos asumir que hay dos premisas: 1) si todo ser es $\mathrm{T}$; 2) y si no todo ser es $\mathrm{T}$, entonces es cierto que cualquier ser es $\mathrm{F}$ o X:

$$
\mathrm{T} \cap \neg \mathrm{T} \Rightarrow F \cup \mathrm{X}
$$

Mediante los pasos posibles de la derivación (negación, conjunción, disyunción e implicación condicional) deducimos la tabla de verdad final:

Tabla 1.

La verificación final de los modos opositores del ser educativo.

\begin{tabular}{llll}
\hline F & T & X & Resultado \\
\hline v & V & v & v \\
v & V & f & v \\
v & f & v & V \\
v & f & f & v \\
f & v & v & v \\
f & V & f & v \\
f & f & v & v \\
f & f & f & v \\
\hline
\end{tabular}

Fuente de datos: Investigación propia

La tabla de verdad es correcta y, por tanto, la contradicción de dos seres (de un conjunto) adquiere un significado verdadero, que consiste en la presencia de la disonancia cognitiva en la intersección del ser-ahí y del ser en línea, que, según la encuesta, se explica por la importancia 
de la inteligencia emocional para adiaforizar las barreras comunicativas en la relación sujetivoobjetiva (hombre-máquina). Sin embargo, la cuestión de las estrategias para incrementar la inteligencia emocional en la educación digital se queda abierta, porque hoy la conciencia digital de los estudiantes está dominada por el factor de espontaneidad y brusquedad provocada por la pandemia del Covid-19, y por tanto parametriza las condiciones de la autoidentificación humana como homo ciberneticus en un contexto particular, sin permitir considerar el objeto de la investigación en un rango más amplio.

La encuesta creada por el grupo de la investigación tuvo como objetivo la obtención de los datos empíricos de la "vida real" en el espacio digital, lo que facilitará el análisis y procesamiento de la información ante los problemas que existen en la educación digital. Habiendo estudiado un número significativo de las fuentes, hemos señalado previamente la importancia de la inteligencia emocional, cuyo funcionamiento es necesario durante el aprendizaje en línea. El experimento involucró a los profesores y los estudiantes de la Universidad de Kyiv Borys Grinchenko, que desde el 12 de marzo del año 2020 hasta el final del año escolar estaban en la cuarentena por la pandemia del Covid-19. Esta razón espontánea fomentó la digitalización de todo el proceso educativo. Por lo tanto, la primera pregunta planteada por el equipo de la investigación fue una duda hipotética: ¿se verá obstaculizada la objetividad de los resultados por el factor de la cuarentena que llevó la digitalización en gran parte forzada, la cual, como muestran los resultados de otros científicos, parametrizó la educación a las condiciones llamadas autoaislamiento? Y lo último, a su vez, ha provocado un estrés académico, la depresión e incluso la implementación del término "fatiga de zoom" como una enfermedad psicológica que se manifiesta por la pérdida de energía debido a las multitareas de videoconferencias, lo que puede hacer concentrarse excesivamente, controlar cada movimiento y preocuparse cómo esto puede ser interpretado por los interlocutores. Sin embargo, la estrategia y la misión de la Universidad de Kyiv Borys Grinchenko adiaforiza estas dudas. Primero, la universidad tiene un sistema digital extenso y bien establecido, cuya implementación y desarrollo había empezado mucho antes de la pandemia. Así, la ética corporativa de los profesores y los estudiantes apegados a la digitalización está orientada al uso activo de las TIC en la educación. En particular, esto se refiere al sistema educativo Moodle, conectado a través de los correos electrónicos corporativos creados para los profesores y los estudiantes por el Departamento de la Informatización de la Universidad. Así, enfatizamos en que el aprendizaje combinado que se realiza mediante la disponibilidad obligatoria de un curso relevante en el sistema Moodle y tiene en cuenta tanto el aprendizaje presencial como el cumplimiento de una parte de trabajo en el curso electrónico ya está disponible en la Universidad de Kyiv Borys Grinchenko durante mucho tiempo. La generación de los estudiantes modernos a priori es una generación digital, lo que está acondicionado por las realidades del siglo XXI, y, a su vez, supone la presencia y el desarrollo de una característica importante para la digitalización: la flexibilidad como el factor de la adaptación rápida a lo que nombramos el ser en línea. Por lo tanto, dada la contribución de la universidad a la educación digital mucho antes de la pandemia, los estudiantes de la Universidad de Kyiv Borys Grinchenko ya tenían un grado significativo de la preparación para la educación digital a distancia.

Todo lo anterior mencionado determinó la exactitud y la objetividad de las respuestas de los encuestados. La pandemia, a su vez, como otros elementos, es explicada, en particular por nuestro equipo de la investigación, desde el punto de vista del concepto filosófico del "Cisne Negro" que define los factores sociales como espontáneos pero tales que pueden ser clarificados e implementados lógicamente (Taleb, 2010).

Notamos que la importancia de la inteligencia emocional en general y en la educación en particular es natural: hoy tenemos una gran cantidad de los estudios en la neuropsicología, anatomía, lingüística y otras ciencias, lo que a priori aumenta su importancia en la educación digital. Sin embargo, en este último caso, tenemos un sistema de la investigación más complicado, ya que el objeto traspasa a una dicotomía hombre-máquina, lo que cambia significativamente el sistema de valores humanos, así como los valores y las funciones de la educación.

\section{Conclusiones}

De tal modo, la investigación tuvo como el objetivo identificar las funciones y la importancia del funcionamiento de la inteligencia emocional en la educación digital. Sin embargo, dada la orientación tecnogénica del sistema de valores de la humanidad, la educación digital, sus presuposiciones y factores fueron 


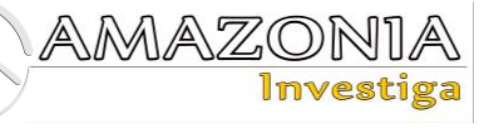

considerados como una parte evolutivamente prevista del todo, que denominamos el ser en línea. Lo último, según nuestras observaciones, es una transformación regular del ser-ahí, pero al mismo tiempo es su contradicción principal, porque la vida actual, asi como la existencia en el tiempo real, en el ser en línea es disonante al repensar el paradigma existencial en los niveles estructural y fenomenológico. Los cambios tan significativos en el paradigma ontológico hacen que se preste más atención a la importancia del funcionamiento de la inteligencia emocional como el sustrato que determina una adaptación positiva a las formas innovadoras de la autoidentificación humana.

Con el motivo de analizar y explicar el núcleo del problema del funcionamiento de la inteligencia emocional en la educación digital, nuestro equipo de la investigación realizó una encuesta empírica de los participantes en el proceso educativo (los profesores y los estudiantes). Esto nos permitió derivar un modelo hipotético-deductivo de la percepción del ser en línea en sus formatos diversos. El modelo, de acuerdo con los resultados del enfoque lógico-praxiológico y de la metodología correspondiente, consiste precisamente en la contradicción entre el ser-ahí determinado físicamente y el ser en línea determinado tecnológicamente, donde la concepción principal del último, que es el infinito digital y la finitud fisica, es la causa del énfasis excesivo del sistema nervioso central de los humanos en el proceso de la adaptación. Bajo tales realidades, la inteligencia emocional se localiza en la intersección de la "reconocibilidad" del hombre y su "disposición ontológica" al mismo tiempo, lo que se agrava especialmente en el ser en línea. La razón de esto es la "captación explícita de la movilidad determinante de la vida actual", que transgrede del Dasein al deadline, lo cual se valida por la presencia de los límites concretos del tiempo.

Sin embargo, como mostraron los resultados del experimento, una función inherente, según los encuestados, para todas las herramientas TIC es la presencia de la comunicación. Y en este caso estamos hablando de la comunicación en general, es decir, tanto de tipo síncrono como asincrónico de la comunicación digital, y por tanto, dada la validez de la comunicación en diferentes tipos de las tareas educativas digitales, donde la comunicación se explica como un acto de la comprensión y empatía entre los comunicantes para adiaforizar cualquier barrera, la inteligencia emocional se supone inmanente como una condición necesaria de la efectividad de la educación digital.
La contradicción entre el ser-ahí y el ser en línea, como el núcleo problemático del funcionamiento de la inteligencia emocional en la educación digital, se verifica mediante el principio de explosión - la fórmula de la lógica clásica e intuicionista según la cual ambos seres fueron extrapolados al conjunto explicado como verdadero y falso al mismo tiempo. Es decir, uno que periódicamente está en disonancia cognitiva debido al problema de la autorregulación psicológica y cognitiva y la transmisión digital de los sentimientos humanos (en el nivel de las relaciones sujetivo-objetivos entre el hombre y la máquina). La verificación de la tabla de verdad confirma la contradicción de dos seres (de un conjunto) y obtiene el significado verdadero, que consiste en la existencia de la disonancia cognitiva en la intersección del ser-ahí y del ser en línea, lo que es extrapolado principalmente a la misión y a la visión de la educación digital como un fundamento de la evolución en el mundo.

\section{Referencias Bibliográficas}

Bar-On, R. (1988) The Development of a Concept of Psychological Well-being. (Unpublished doctoral dissertation) Rhodes University South Africa, http://vital.seals.ac.za:8080/vital/access/man ager/Repository/vital:2928?site_name=Glob alView

Boyatzis, R.E. \& Goleman, D. (2001) Emotional Competence Inventory - University Version. Self-assessment Questionnaire, Profile and Interpretive Notes Hay Resources Direct London.

Buzdar, M. A., Ali, A., \& Tariq, R. U. H. (2016). Emotional intelligence as a determinant of readiness for online learning. International Review of Research in Open and Distributed Learning, 17(1), 148-158.

Chandra, Y. (2020). Online education during COVID-19: perception of academic stress and emotional intelligence coping strategies among college students. Asian Education and Development Studies, 10(2), pp. 229-238

Conte, J.M. (2005) A review and critique of emotional intelligence measures Journal of Organizational Behaviour 26 433-440

Damasio, A.R. (1994) Descartes' Error: Emotion, Reason and The Human Brain. New York: Harper Collins.

Davies, A., \& Fidler, D. et al (2011). Future Work Skills 2020. Institute for the Future for University of Phoenix Research Institute. Retrieved from: https://www.iftf.org/uploads/media/SR- 
1382A_UPRI_future_work_skills_sm.pdf (accessed October2020).

Davis, M., Stankov, L. \& Roberts, R. (1998) Emotional intelligence: In search of an elusive construct. Journal of Personality and Social Psychology, 75, 989-1015

Dos Reis, A. (2015). To Be a (Blended) Teacher in the 21st Century-Some Reflections. International Journal of Research in Elearning, 1(1), 11-24.

DQ Global Standards Report (2019). World's first global standard for digital literacy, skills and readiness launched by the Coalition for Digital Intelligence. Retrieved from: https://www.dqinstitute.org/ (accessed July 2020).

Heidegger, M., \& Hofstadter, A. (1988). The Basic Problems of Phenomenology, Revised Edition (Studies in Phenomenology and Existential Philosophy) (Revised ed.). Indiana University Press.

Humphrey, N., Curran, A., Morris, E., Farrell, P. \& Woods, K. (2007) Emotional Intelligence and Education: A critical review, Educational Psychology, 27(2), 235-254, DOI: 10.1080/01443410601066735

Hymes, D.H. (1972). Communicative competence. In Pride, J.B., \& J. Holmes, J. (Eds.) Sociolinguistics: selected readings. (pp. 269-293). Harmondsworth: Penguin.

Keefer, K., Parker, J., \& Saklofske, D. (2018). Emotional intelligence in education. Integrating research with practice. Cham, Switzerland: Springer.

Makhachashvili, R., Semenist, I., \& Bakhtina, A. (2020a). Digital Skills Development and ICT Tools for Final Qualification Assessment: Survey Study for Students and Staff of European and Oriental Philology Programs. Electronic Scientific Professional Journal "OPEN EDUCATIONAL EENVIRONMENT OF MODERN UNIVERSITY”, (9), 54-68. https://doi.org/10.28925/2414-0325.2020.9.5
Makhachashvili, R., Semenist, I., \& Bakhtina, A. (2020b). ICT Tools and Practices for Final Qualification Assessment in the Framework of COVID-19 Lockdown. Innovative Educational Technologies, Tools and Methods for E-learning Monograph. University of Silesia in Katowice, Poland, Katowice, pp. 183-194.

Makhachashvili, R., Semenist, I., Shtaltovna, Y. \& Bakhtina, A. (2021c). Soft Skills and ICT Tools for Final Qualification Assessment in Universities of Ukraine and India in COVID19 Framework. Psychology and Education Journal, 58(2), 849-861. https://doi.org/10.17762/pae.v58i2.1959

Radford, M. (2006) Book Review: A Passion for Teaching. Theory and Research in Education, 4(2), pp. 227-229.

Restian, A. (1981). Homo ciberneticus. Scientific and Encyclopedic Publishing House.

Taleb, N. N. (2010). The Black Swan: Second Edition: The Impact of the Highly Improbable: With a new section: "On Robustness and Fragility" (Incerto) (2nd ed.). Random House Trade Paperbacks. https://www.penguinrandomhouse.com/book s/176226/the-black-swan-second-edition-bynassim-nicholas-taleb/

Van Deursen, A. J., Bolle, C. L., Hegner, S. M., \& Kommers, P. A. (2015). Modeling habitual and addictive smartphone behavior: The role of smartphone usage types, emotional intelligence, social stress, self-regulation, age, and gender. Computers in human behavior, 45, 411-420.

Weber, M., Gerth, H. H., \& Mills, W. C. (1958). From Max Weber: Essays in Sociology. Oxford University Press (Galaxy imprint).

Zahed-Babelan, A., \& Moenikia, M. (2010). The role of emotional intelligence in predicting students' academic achievement in distance education system. Procedia-Social and Behavioral Sciences, 2(2), 1158-1163. 